\title{
Objective correlates and determinants of bicycle commuting propensity in an urban environment
}

T. Cole-Hunter,

D. Donaire-Gonzalez, a, , ,

A. Curto,

A. Ambros,

A. Valentina,

J. Garcia-Aymericha, b,

D. Martínez,

L.M. Braune,

M. Mendez,

M. Jerrett ${ }^{\text {, }}$,

D. Rodriguez,

A. de Nazelle,

M. Nieuwenhuijsen ${ }^{a, b}$

${ }^{a}$ Centre for Research in Environmental Epidemiology, Barcelona, Spain

${ }^{\circ}$ CIBER Epidemiología y Salud Pública (CIBERESP), Barcelona, Spain

' Department of Physical Activity and Sports Sciences, Fundació Blanquerna, Barcelona, Spain

d Departament de Ciències Experimentals i de la Salut, Universitat Pompeu Fabra, Barcelona, Spain

' Department of City \& Regional Planning, University of North Carolina, Chapel Hill, USA

' Department of Nutrition, Gillings School of Global Public Health, University of North Carolina, Chapel Hill, USA

${ }^{9}$ Department of Environmental Health Sciences, University of California, Los Angeles, USA

${ }^{\mathrm{h}}$ Centre for Environmental Policy, Imperial College London, London, United Kingdom

doi:10.1016/j.trd.2015.07.004

Highlights

-We performed a travel survey in the Mediterranean city of Barcelona, Spain.

- Our study recruitment design surveyed a relatively-high proportion of bicyclists.

-We ascertained urban environmental determinants for bicycle commuting using GIS.

- Bicycle sharing stations and greenness were motivators for bicycle commuting.

- Public transport stations and elevation were deterrents for bicycle commuting.

\section{Abstract}

Objective 
Bicycle use for commuting is being encouraged not only to address physical inactivity, but also vehicular congestion, air pollution and climate change. The current study aimed to ascertain the urban environmental correlates and determinants of bicycle use for commuting (bicycle commuting) among the working or studying population in Barcelona, Spain.

\section{Methods}

Adults ( $n=769 ; 52 \%$ females) recruited whilst commuting within Barcelona (Spain) responded to a comprehensive telephone survey concerning their travel behaviour. Based upon responses collected from June 2011 to May 2012, participants were categorised into four groups: frequent bicyclists, infrequent bicyclists, willing non-bicyclists, and unwilling non-bicyclists. The determinants of frequency and willingness (propensity) to commute by bicycle were assessed by multinomial logistic regression models adjusted for potential confounders and covariates.

\section{Results}

The number of public bicycle stations surrounding the home address and amount of greenness surrounding the work/study address were significant positive determinants of bicycle commuting propensity. On the other hand, the number of public transport stations surrounding the home address and elevation of the work/study address were significant negative determinants of bicycle commuting propensity. Individual age, education level, gender, nationality, physical activity level and commute distance significantly affected this propensity.

\section{Conclusion}

Greater availability of public bicycle stations and higher levels of urban greenness may increase bicycle use by adults commuting within a city such as Barcelona, Spain. Electrically-assisted public bicycles may address the challenge of elevation, making this system a more competitive mode against traditional motorised public transport.

\section{Abbreviations}

- $d B(A)$, A-weighted decibels;

- GIS, geographic information system;

- NDVI, Normalized Difference Vegetation Index;

- $\mathrm{NO}_{2}$, nitrogen dioxide;

- RBA, route-by-area 
Keywords

- Commute;

- Bicycle;

- Urban;

- Environment;

- Greenness;

- Elevation

\section{Introduction}

Physical activity is associated with myriad mental and physical health benefits (Haskell et al., 2007 and U.S. Department of Health and Human Services, 1996). Insufficient physical activity may result in cardio-metabolic conditions such as obesity, diabetes, and high blood pressure (Furie and Desai, 2012) which rank as leading causes of death and disability globally (Lim et al., 2012). An urban environment can facilitate the incorporation of regular physical activity into daily routines (Brown et al., 2013), such as with active transport (Pucher et al., 2010), increasing physical activity levels. Moreover, a substantial population shift from motorised to active transport modes may reduce emissions and improve air quality, while also reducing traffic accidents, bringing greater environmental and public health benefits (De Nazelle et al., 2011).

Most cities have only a very small proportion of commuting trips by bicycle (Pucher et al., 2010), and our study site of Barcelona is no exception with only $1.3 \%$ of the trips being made by bicycle (Autoritat del Transport Metropolità, 2013). However, other cities have a much higher bicycle share. For example, Copenhagen has approximately a third of all commuting trips by bicycle (City of Copenhagen, 2012). While recently more studies are being conducted in non-exceptional cities, the majority of previous studies have focused on exceptional cities like Copenhagen, and arguably, determinants of bicycling may vary at different levels of participation in the population. For example, high levels of participation in bicycling may reduce the individual risk of bicyclists by heightening awareness of their presence and thereby mitigating interaction with motorised traffic (Bhatia and Wier, 2011). While participation rates are still low, however, bicycle infrastructure such as separated bike lanes on major roads may reduce the risks for bicyclists and encourage participation by offsetting the negative effects of higher traffic volumes (Broach et al., 2012, Wegman et al., 2012 and Winters et al., 2010) and by enhancing connectivity and 
travel times (Winters et al., 2011). The vast majority of studies examining the determinants of bicycling commuting have focused on infrastructure features such as this in different cultures and geographies (Panter and Jones, 2010, Molina-García et al., 2010, Rodríguez and Joo, 2004, Titze et al., 2008 and Troped et al., 2001). One potential reason for this focus is the prevailing framework for understanding travel behaviour. Bicycling infrastructure and other built environment attributes can be thought as influencing travel behaviour by affecting the relative utility of different travel modes (Boarnet and Crane, 2001, Cervero, 2002 and Crane, 1996). The utility of a given mode is related to the perceived cost and difficulty of using that mode (Boarnet and Crane, 2001, Cervero, 2002 and Handy and Boarnet, 2002). By influencing travel times, convenience, safety, pleasantness, and so on, infrastructure features have a direct influence on the choice of different travel modes.

Despite the emerging importance of the built environment for bicycling, several measurement improvements are necessary. For one, a broader set of environmental measures is required. Some built environment attributes have been less consistently covered than others in the existing literature, such as greenness and noise, which are features of a typical urban environment that warrant consideration. Based on the travel behaviour framework, greenness and noise likely affect the utility of bicycling by influencing the overall pleasantness and enjoyability of it. While noise may directly influence utility in this way (Winters et al., 2011 and Panter et al., 2008), greenness may generally promote physical activity (James et al., 2015, Lee et al., 1999, Rodriguez et al., 2014, Frank et al., 2007 and Ewing et al., 2008). In fact, greenness has been observed as one of the most stimulating perceived (subjective) environmental determinants to bicycle commute (Wahlgren and Schantz, 2012). In addition to a broader set of environmental measures being required, the context in which built environment measures are made deserves attention. Most studies have focused on the environment around where trips begin. Yet, the attributes of destinations and of the route in-between are also likely to impact the utility of a given mode. For example, Winters and associates (Winters et al., 2011) find that features of the origin, route, and destination environments are all significant determinants of bicycling and in different ways (e.g., some determinants are more important in one spatial zone than the others). Not only can the attributes of the built environment be important for mode choice, but the perception of travellers about these attributes can be too. In the end, the utility of a given mode for a person is a melding of the actual attributes, the 
traveller's perceptions of those attributes, and the traveller's valuation of the importance of those attributes. Indeed, studies have considered both objective and perceived measures of bicycling infrastructure, with interesting comparisons. Previously, perceptions have been found to be more important predictors of travel behaviour than objective measures of the same environmental characteristics (Dill and Voros, 2007 and Hoehner et al., 2005). McGinn and associates (McGinn et al., 2007) found both to be significant, but varying in magnitude. Both objective and perceived measures of the built environment are likely to factor into the valuation of travel modes and resulting decisions about how to travel.

While attitudes and perceptions of an individual are known to be important theoretical considerations for travel planning (Dill et al., 2014, Sener et al., 2009a and Sener et al., 2009b), a review on the determinants of bicycle commuting highlighted gaps in the literature and the need to gather more evidence on objective and built environmental determinants (Heinen et al., 2010). In this paper we address this call by focusing on an expanded set of built environment measures calculated in different contexts (around home, around work/study, and along the route). Specifically, we aim to (1) describe the urban environmental characteristics (including greenness and noise) of three different spatial zones (home, work/study, and commute

route areas) according to a participant's propensity to bicycle commute; and (2) evaluate the strength of the relationship between multiple yet specific urban environmental determinants of bicycle commuting (including greenness and noise) and the propensity to bicycle commute.

\section{Methods}

\section{Study design}

The current study design was cross-sectional, utilising a geographic information system (GIS) to characterize the built environment at the participant's home and work or school address area, as well as the commute route area between the two addresses. Data on participant commute behaviour in the study location was collected with a travel survey.

\section{Study location}

Barcelona is a Mediterranean city on the north-eastern coast of Spain with a population of 1.6 million. The city is densely populated with approximately 16,000 inhabitants and 6000 vehicles per $\mathrm{km}^{2}$ (Departament d'Estadística, 2011). Bicycle use in the city is 
accommodated by several location features, including temperate climate, relatively flat topography and a public bicycle sharing program. This program (Bicing) was introduced in 2007 with a network density of 3.7 bicycles per 1000 inhabitants with stations evenly distributed by distance throughout the city, resulting in a $36 \%$ increase in daily bicycle trips since the inception of the program ( Desarrollo Organización Movilidad, 2011 and Midgley, 2011). Despite Bicing, bicycling is still a very minor mode of transport at $1.3 \%$ of modal share; the public transport network has a share of $19 \%$, and private motor vehicles have $31 \%$, with the rest being walking ( Autoritat del Transport Metropolità, 2013).

\section{Participant recruitment}

Due to low modal share of bicycles for commuting we chose to recruit participants on the street to be able to identify and target bicyclists for obtaining sufficient analytical power, while also recruiting non-bicyclists as users of other travel modes. Forty locations throughout the city of Barcelona were used to recruit participants between June 2011 and May 2012. These 40 points resulted from randomly selecting four locations to sample within each of the 10 city districts (see Appendix Figs. A and B), thereby providing adequate coverage of the city. Nearby locations were chosen as points of recruitment for targeted participants representing various commute modes. For example, at the 40 points, we recruited bicycle commuters near Bicing stations, public transport users near public transport stations, and private transport users near vehicle parking lots. Each point was sampled by three trained interviewers between 7:45 and 11:30 a.m. during four days within a randomly selected week. When first approached, potential participants were given an initial screening questionnaire to determine whether they were suitable to be surveyed, namely of being (physically) capable of riding a bicycle for commuting purposes in Barcelona. Once recruited, participants responded to a travel survey by Computer Assisted Telephone Interviewing (CATI; QDS 2.0, NOVA Research Company) at a later date. To be included in the analysis of this study, they were required to meet the following inclusion criteria of: (i) being an adult of 18 years or more; (ii) having lived in Barcelona city since at least 2006 (allowing adequate commuting experience); (iii) working or studying in Barcelona city; (iv) being healthy enough to ride a bicycle unassisted for $20 \mathrm{~min}$; $(\mathrm{v})$ having a commute distance of greater than one $\mathrm{km}$ (or 10 min duration) from home to work or school; (vi) having a maximum distance of $400 \mathrm{~m}$ (or five minutes duration) from home to the first transport mode; (vii) using at least one mode of transport other 
than walking within the commute, and; (viii) having a geocodable home and work/study address. The study protocol was approved by the Clinical Research Ethical Committee of the Parc de Salut Mar (CEICParc de Salut Mar), and written informed consent was obtained from all participants before participation.

\section{Participant data collection}

The travel survey, which took approximately 30 minutes to complete, consisted of both previously-established questions adapted from their original source and original questions designed specifically for this study. All questions were tested for local applicability and comprehension in a convenience sample of 36 participants prior to the main sampling period. The information collected in the survey included: physical activity level (Craig et al., 2003), bicycle use (Forsyth and Krizek, 2012), common use of transport modes (Federació de Municipis de Catalunya, 2008), home and work or school addresses and socio-demographic variables.

Participant commute environment characteristics were estimated by geocoding the home and work/school addresses with ArcGIS software (v10.0; Environmental Systems Research Institute Inc., Redlands CA, 2010). The micro-environments of the home and work/study address area were assessed within the GIS environment using a buffer of $400 \mathrm{~m}$ radius. This magnitude of radius was chosen to reflect the distance considered reasonable to be walked in five minutes (at the average walking speed of adults, $4.8 \mathrm{~km} / \mathrm{h}$ ) in compliance with the sixth inclusion criteria for participation. To estimate the likely route a participant would take for their commute, the 'shortest route' was calculated using ArcGIS Network Analyst, considering the distance as impedance and all streets as bi-directional. As bicyclists may not necessarily choose the shortest route, micro-environments of the smallest rectangle enclosing the route [i.e. a buffer envelope of 'routeby-area' (RBA); see Fig. 1] were assessed. 


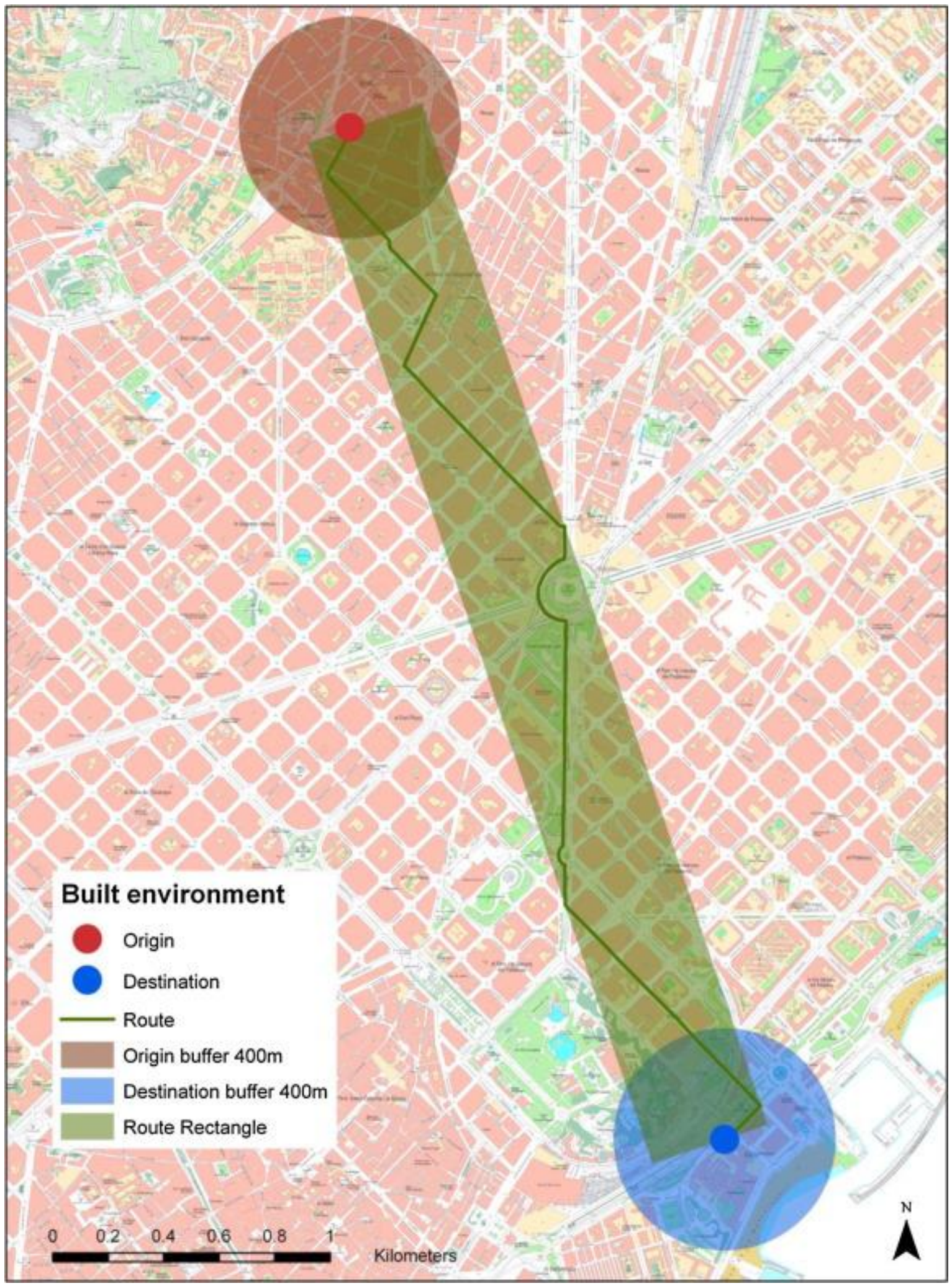

Fig. 1.

'Route-by-area' buffer envelope within GIS environment. Adults ( $n=769 ; 52 \%$ females) commuting to work or school in Barcelona responded to a comprehensive telephone survey concerning their travel behaviour through June 2011 to May 2012. To estimate the likely route a respondent would take, the 'shortest route' was calculated using ArcGIS Network Analyst, considering the distance as impedance and all streets as bi- 
directional (so as not to discriminate against non-road-bound commute modes such as bicycling). To address the uncertainty of the calculated path, environment attributes associated with commute routes were calculated using the smallest rectangle enclosing the route (that is the buffer envelope of 'route-by-area', RBA). Commute route attributes, such as noise and nitrogen dioxide $\left(\mathrm{NO}_{2}\right)$ levels, slope, greenness index, and bikeability index, were calculated and collated according to the individual RBA envelope of each respondent.

\section{Objective environmental determinants}

As previously noted, in addition to the influence of mode choice attributes such as time and cost of travel, the built and natural environments can be considered for understanding mode choices such as to go by bicycle (Rodríguez and Joo, 2004). Thus, the following determinants were considered to address some knowledge gaps in the field (Heinen et al., 2010). Environmental characteristics of each home and work/study area, and RBA, were calculated, including noise (daytime) and nitrogen dioxide $\left(\mathrm{NO}_{2}\right)$ levels, elevation, greenness, bicycle lanes, bicycle racks, presence of Bicing (public bicycle) and public transport stations. Noise was calculated as a mean and percentiles in $\mathrm{dB}(\mathrm{A})$ level equivalent (LAeq) modeled using measured noise and transit data from Barcelona's strategic noise map developed in the year 2007 ( Ajuntament de Barcelona, 2010) based upon previous work ( Ministere de L'Environnement, 1980); briefly, the model considers: the number of light vehicles, the number of heavy vehicles, the speed of circulation, the street slope, the type of pavement of the street, and reflections of the building on either side of the road. The noise variable used for analyses was the proportion of street length above a $55 \mathrm{~dB}(\mathrm{~A})$ threshold ( World Health Organisation, 2011). $\mathrm{NO}_{2}$ was calculated as a mean and percentiles using a land-use regression model developed for a previous project (Beelen et al., $\underline{2013}$ ) in grid cells of 10 square-metres. Elevation was calculated with a Digital Elevation Model ( Beyer, 2012). Greenness was calculated as a mean and percentiles in Normalized Difference Vegetation Index (NDVI) via satellite imagery (LANDSAT 4 and 5, NASA). For NDVI, a pixel resolution of $30 \mathrm{~m}$ was used from the month of April which displayed the most developed amount of 'greenness'. Also measured were bicycle parking and lanes digitised from existing maps ( Ajuntament de Barcelona, 2011). Bicing (IMI - Informació de Base i Cartografia) and public transport stations ( Generalitat de Catalunya, 2011) were counted within home and work/study areas. 
We grouped our respondents according to their propensity to bicycle commute. This was based on logic in their responses; that they considered themselves generally to use the bicycle often or not and that they used it often or not at the time (reflected by the number of trips by bicycle in the week prior) of responding to the survey. Participants were first classified as 'bicycle commuters' or 'non-bicycle commuters'. A 'bicycle commuter' indicated bicycling as at least "sometimes" their general transport mode and bicycle commuted in the week prior to survey administration, while a 'non-bicycle commuter' indicated bicycling as "never or nearly never" their general transport mode and did not bicycle commute in the week prior to survey administration.

Bicycle commuters were further classified as either 'frequent' or 'infrequent'. A 'frequent' bicycle commuter indicated bicycling as "often" (or more frequently) their general transport mode and bicycle commuted for four or more days in the week prior to survey administration (i.e. above the total group median). An 'infrequent' bicycle commuter, however, indicated bicycling as "sometimes" (or less frequently) their general transport mode and/or bicycle commuted at least once in the week prior to survey administration. For example, if a participant responded that they "never or nearly never" bicycled for transport but performed at least one bicycle trip in the week prior to survey administration then they were considered an infrequent bicycle commuter; if no bicycle trips were performed then they were considered a non-bicycle commuter.

Non-bicycle commuters were further classified as either 'willing' or 'unwilling'. A 'willing' non-bicycle commuter indicated bicycling as "never or nearly never" their general transport mode but indicated that they would consider bicycle commuting in Barcelona by answering positively to "considering costs, travelling time, comfort and safety, how ready would you be to use the bicycle/Bicing for your trip to work or educative centre?" An 'unwilling' non-bicycle commuter indicated bicycling as "never or nearly never" their general transport mode, and indicated that they would not consider bicycle commuting in Barcelona by answering negatively to the above.

\section{Data analyses}

All data analyses were performed using STATA/SE (v12.0, StataCorp LP, Texas USA). Continuous data was tested for normality with Shapiro-Wilk and for significance $(p<0.05)$ with either ANOVA or Kruskal-Wallis (according to distribution of variable) across the four 
bicycle commuting propensity groups. Categorical data was observed via cross-tabulations and tested for significance with chi-squared or Fisher chi-squared (according to class and distribution of variable) across bicycle commuting propensity groups.

Objective environmental measures (described earlier as the main exposures) that may determine bicycle commuting propensity were evaluated using a multinomial logistic regression model to produce relative risk ratios and $95 \%$ confidence intervals. Participant characteristics were dichotomized before testing as potential confounders, including gender, nationality (Spanish/non-Spanish), education level ( $<l \geqslant$ tertiary e.g. university), and bicycle access (yes/no; 'yes' as reported either private household ownership or public Bicing membership). Age and calculated commute distance were kept as continuous variables and also tested as potential confounders. They were included in the final model as confounders if (separately) statistically significantly-associated to the outcome or if they changed the main predictor of interest by $10 \%$ or more. Personal determinants such as gender were considered in the model as they are known to influence preference for environmental features ( Garrard et al., 2008). Commute distance was also included in the model as it has been seen as a key determinant (travel cost) of bicycle commuting ( Broach et al., 2012, Hood et al., 2011 and Ortúzar et al., 2000). Ultimately, objective determinants of bicycle commuting propensity were highlighted in the model by gradual removal of non-confounder variables (or variables of interest, e.g. noise or greenness) according to insignificance probability in any of the three propensity outcome categories. This was done using a supervised (manual), stepwise (backwards) elimination regression process. Population density, as an indicator of urban centres and infrastructure hubs, at the home and work/study address (400 m buffer radii) area was tested with sensitivity analysis of the final model. Final model fitness was checked with a (post-estimation) generalized Hosmer-Lemeshow goodness-of-fit test. Unwilling non-bicyclists were used as the base for comparison in this model to indicate the increased likelihood of bicycling or being willing to bicycle.

Additionally, as previous research suggests student behaviour may be likely different compared to non-students (workers) Heinen et al., 2010, a supplemental analysis was performed stratifying this final model by occupational status (worker/student).

\section{Results}

Description of participant and environmental correlates 
In total 18,469 people were systematically approached across the forty sampling points of Barcelona city between June 2011 and May 2012. Of those, 6701 participants accepted to answer initial screening questions, 1406 participants fulfilled the inclusion criteria and 769 participants completed the travel survey by phone. Included participants had an approximate mean age of 37 , were of equivalent gender proportion, and in the normal range of body mass index. The majority were national (Spanish) and in a professional occupation (i.e. not students). Statistically significant differences existed between subgroups of bicycle commuting propensity. Personal determinants of age and occupational status, however, were not significantly different between these sub-groups. Relative to other respondents frequent bicycle commuters were more likely to: be male; have access to a bicycle; have a cheaper and shorter commute; and have a universitylevel education or equivalent (Table 1).

\section{Table 1.}

Participant characteristics as a total group and according to propensity for bicycle commuting.

\begin{tabular}{|c|c|c|c|c|c|c|}
\hline Descriptive & $\begin{array}{l}\text { All } \\
n=769\end{array}$ & $\begin{array}{l}\text { Bicyclist, } \\
\text { Frequent } \\
n=281\end{array}$ & $\begin{array}{l}\text { Bicyclist, } \\
\text { Infrequent } \\
n=120\end{array}$ & $\begin{array}{l}\text { Non- } \\
\text { bicyclist, } \\
\text { Willing } \\
n=141\end{array}$ & $\begin{array}{l}\text { Non- } \\
\text { bicyclist, } \\
\text { Unwilling } \\
n=227\end{array}$ & $\begin{array}{l}p- \\
\text { value }^{(a b)}\end{array}$ \\
\hline Age, years [mean(SD)] & $\begin{array}{l}36.6 \\
(10.4)\end{array}$ & $\begin{array}{l}35.5 \\
(9.78)\end{array}$ & $\begin{array}{l}35.7 \\
(9.79)\end{array}$ & $\begin{array}{l}37.3 \\
(11.4)\end{array}$ & $\begin{array}{l}37.9 \\
(10.7)\end{array}$ & $0.374^{a}$ \\
\hline $\begin{array}{l}\text { Gender [Female; \# } \\
(\%)]\end{array}$ & 51.8 & 44.5 & 43.3 & 51.1 & 65.6 & $<0.001^{\underline{b}}$ \\
\hline $\begin{array}{l}\mathrm{BMI}[\mathrm{kg} / \mathrm{m3} ; \\
\text { mean(SD)] }\end{array}$ & $\begin{array}{l}23.5 \\
(3.31)\end{array}$ & $\begin{array}{l}23.3 \\
(3.14)\end{array}$ & $\begin{array}{l}23.4 \\
(3.17)\end{array}$ & $\begin{array}{l}23.8 \\
(3.56)\end{array}$ & $\begin{array}{l}23.6 \\
(3.41)\end{array}$ & $0.325^{a}$ \\
\hline $\begin{array}{l}\text { Nationality [Spanish; \# } \\
(\%) \text { ] }\end{array}$ & 87.4 & 86.8 & 80.8 & 85.8 & 92.5 & $0.015^{\underline{b}}$ \\
\hline $\begin{array}{l}\text { Occupation [Student; \# } \\
(\%)]\end{array}$ & 13.0 & 11.4 & 15.8 & 17.7 & 10.6 & $0.141^{\underline{b}}$ \\
\hline $\begin{array}{l}\text { Household income, } \\
\text { monthly [1-5 k€; } \\
\text { mean(SD)] }\end{array}$ & $\begin{array}{l}1.99 \\
(1.22)\end{array}$ & $\begin{array}{l}1.97 \\
(1.21)\end{array}$ & $\begin{array}{l}2.03 \\
(1.26)\end{array}$ & $\begin{array}{l}1.89 \\
(1.07)\end{array}$ & $\begin{array}{l}2.03 \\
(1.26)\end{array}$ & $0.004^{b}$ \\
\hline $\begin{array}{l}\text { Bicycle access, } \\
\text { household/Bicing [Yes; } \\
\#(\%)]\end{array}$ & 74.9 & 100 & 100 & 59.6 & 40.1 & $<0.001^{b}$ \\
\hline $\begin{array}{l}\text { Bicycle trips, weekly } \\
(\#) \text { [mean(SD)] }\end{array}$ & $\begin{array}{l}3.64 \\
(1.81)\end{array}$ & $\begin{array}{l}4.84 \\
(0.56)\end{array}$ & $2.6(1.01)$ & $0(0)$ & $0(0)$ & $<0.001^{\underline{b}}$ \\
\hline $\begin{array}{l}\text { Commute days, } \\
\text { weekly (\#) [mean(SD)] }\end{array}$ & $\begin{array}{l}4.85 \\
(0.59)\end{array}$ & $\begin{array}{l}4.91 \\
(0.45)\end{array}$ & $\begin{array}{l}4.73 \\
(0.68)\end{array}$ & $\begin{array}{l}4.87 \\
(0.59)\end{array}$ & $\begin{array}{l}4.83 \\
(0.72)\end{array}$ & $0.065^{a}$ \\
\hline $\begin{array}{l}\text { Commute distance, } \\
\text { estimated }(\mathrm{km}) \\
{[\text { mean }(\mathrm{SD})]}\end{array}$ & $\begin{array}{l}3.85 \\
(2.05)\end{array}$ & $\begin{array}{l}3.19 \\
(1.59)\end{array}$ & $\begin{array}{l}3.78 \\
(1.93)\end{array}$ & $\begin{array}{l}4.32 \\
(2.11)\end{array}$ & $\begin{array}{l}4.42 \\
(2.34)\end{array}$ & $<0.001^{a}$ \\
\hline $\begin{array}{l}\text { Commute duration, } \\
\text { reported (mins) } \\
\text { [mean(SD)] }\end{array}$ & $\begin{array}{l}23.8 \\
(12.0)\end{array}$ & $\begin{array}{l}21.1 \\
(9.09)\end{array}$ & $\begin{array}{l}24.1 \\
(12.3)\end{array}$ & $\begin{array}{l}26.7 \\
(13.3)\end{array}$ & $\begin{array}{l}25.2 \\
(13.4)\end{array}$ & $<0.001^{a}$ \\
\hline Commute expense, & 5.59 & 1.27 & 5.42 & 9.31 & 9.01 & $<0.001^{a}$ \\
\hline
\end{tabular}




\begin{tabular}{|c|c|c|c|c|c|c|}
\hline Descriptive & $\begin{array}{l}\text { All } \\
n=769\end{array}$ & $\begin{array}{l}\text { Bicyclist, } \\
\text { Frequent } \\
n=281\end{array}$ & $\begin{array}{l}\text { Bicyclist, } \\
\text { Infrequent } \\
n=120\end{array}$ & $\begin{array}{l}\text { Non- } \\
\text { bicyclist, } \\
\text { Willing } \\
n=141\end{array}$ & $\begin{array}{l}\text { Non- } \\
\text { bicyclist, } \\
\text { Unwilling } \\
n=227\end{array}$ & $\begin{array}{l}p- \\
\text { value }^{(a b)}\end{array}$ \\
\hline $\begin{array}{l}\text { weekly (€,-fuel) } \\
\text { [mean(SD)] }\end{array}$ & $(8.33)$ & $(5.02)$ & $(10.8)$ & $(8.85)$ & $(7.19)$ & \\
\hline $\begin{array}{l}\text { Vigorous exercise, } \\
\text { weekly [Yes; \# (\%)] }\end{array}$ & 60.1 & 66.2 & 73.3 & 47.5 & 53.3 & $<0.001^{\underline{b}}$ \\
\hline $\begin{array}{l}\text { Chronic disease, } \\
\text { reported [Yes; \# (\%)] }\end{array}$ & 7.54 & 8.19 & 10.8 & 3.55 & 7.49 & $0.154^{b}$ \\
\hline $\begin{array}{l}\text { Tertiary educated } \\
\text { [Yes; \# (\%)] }\end{array}$ & 69.8 & 77.6 & 72.5 & 51.8 & 70.0 & $<0.001^{\underline{b}}$ \\
\hline \multicolumn{7}{|c|}{$\begin{array}{l}\text { a ANOVA/Kruskal-wallis for normally/abnormally continuous variables, respectively. } \\
\text { b Chi-squared for categorical variables. }\end{array}$} \\
\hline \multirow{5}{*}{\multicolumn{7}{|c|}{$\begin{array}{l}\text { Adults ( } n=769 ; 52 \% \text { females) commuting to work or school in Barcelona responded to } \\
\text { a comprehensive telephone survey concerning their travel behaviour through June } 2011 \\
\text { to May 2012. Based upon responses, participants were categorised into four groups: } \\
\text { frequent bicyclists, infrequent bicyclists, willing non-bicyclists, and unwilling non- } \\
\text { bicyclists. Bicycle access is attributed if a participant has a personal bicycle }\end{array}$}} \\
\hline & & & & & & \\
\hline & & & & & & \\
\hline & & & & & & \\
\hline & & & & & & \\
\hline \multicolumn{7}{|c|}{ or Bicing registration or both. Commute distance is length of route estimated with GIS } \\
\hline
\end{tabular}

Table options

In the same respect, the significant environmental correlates of being a frequent bicycle commuter were: higher counts of bicycle racks and public bicycle stations in the home and work/study address area; greater proportion of bicycle lanes in the home and work/study address area and commute route area; and lower elevation in the home and work/study address area. Greenness of the home address area was a significant correlate of being a willing non-bicycle commuter (Table 2). 
Table 2.

Environmental descriptives as a total group and according to propensity for bicycle commuting

\begin{tabular}{|c|c|c|c|c|c|c|}
\hline Descriptive & $\begin{array}{l}\text { All } \\
n=769\end{array}$ & $\begin{array}{l}\text { Bicyclist, } \\
\text { Frequent } \\
n=281\end{array}$ & $\begin{array}{l}\text { Bicyclist, } \\
\text { Infrequent } \\
n=120\end{array}$ & $\begin{array}{l}\text { Non- } \\
\text { bicyclist, } \\
\text { Willing } \\
n=141\end{array}$ & $\begin{array}{l}\text { Non- } \\
\text { bicyclist, } \\
\text { Unwilling } \\
n=227\end{array}$ & $\begin{array}{l}p- \\
\text { value }\end{array}$ \\
\hline \multicolumn{7}{|l|}{ Bicycle racks [mean(SD)] } \\
\hline $\begin{array}{l}\text { Home, count in } 400 \mathrm{~m} \\
\text { buffer }\end{array}$ & $\begin{array}{l}18.7 \\
(15.7)\end{array}$ & $\begin{array}{l}21.9 \\
(15.8)\end{array}$ & $\begin{array}{l}20.2 \\
(16.4)\end{array}$ & $\begin{array}{l}16.1 \\
(15.3)\end{array}$ & $\begin{array}{l}15.6 \\
(14.7)\end{array}$ & $<0.001$ \\
\hline $\begin{array}{l}\text { Work/study, count in } \\
400 \text { m buffer }\end{array}$ & $\begin{array}{l}25.9 \\
(16.8)\end{array}$ & $\begin{array}{l}27.8 \\
(16.4)\end{array}$ & $\begin{array}{l}26.9 \\
(15.7)\end{array}$ & $\begin{array}{l}24.9 \\
(16.6)\end{array}$ & $\begin{array}{l}23.6 \\
(17.7)\end{array}$ & 0.026 \\
\hline \multicolumn{7}{|l|}{$\begin{array}{l}\text { Public bicycle stations } \\
\text { [mean(SD)] }\end{array}$} \\
\hline $\begin{array}{l}\text { Home, count in } 400 \mathrm{~m} \\
\text { buffer }\end{array}$ & $\begin{array}{l}4.24 \\
(2.53)\end{array}$ & $\begin{array}{l}4.80 \\
(2.44)\end{array}$ & $\begin{array}{l}4.58 \\
(2.58)\end{array}$ & $\begin{array}{l}3.69 \\
(2.45)\end{array}$ & $\begin{array}{l}3.71 \\
(2.48)\end{array}$ & $<0.001$ \\
\hline $\begin{array}{l}\text { Work/study, count in } \\
400 \text { m buffer }\end{array}$ & $\begin{array}{l}4.91 \\
(3.11)\end{array}$ & $\begin{array}{l}5.49 \\
(3.08)\end{array}$ & $\begin{array}{l}4.90 \\
(2.85)\end{array}$ & $\begin{array}{l}4.65 \\
(3.41)\end{array}$ & $\begin{array}{l}4.35 \\
(2.96)\end{array}$ & $<0.001$ \\
\hline \multicolumn{7}{|l|}{$\begin{array}{l}\text { Public transport stations } \\
\text { [mean(SD)] }\end{array}$} \\
\hline $\begin{array}{l}\text { Home, count in } 400 \mathrm{~m} \\
\text { buffer }\end{array}$ & $\begin{array}{l}17.2 \\
(5.37)\end{array}$ & $\begin{array}{l}16.4 \\
(4.93)\end{array}$ & $\begin{array}{l}17.0 \\
(5.30)\end{array}$ & $\begin{array}{l}17.6 \\
(5.52)\end{array}$ & $\begin{array}{l}18.0 \\
(5.73)\end{array}$ & $<0.001$ \\
\hline $\begin{array}{l}\text { Work/study, count in } \\
400 \mathrm{~m} \text { buffer }\end{array}$ & $\begin{array}{l}18.3 \\
(7.00)\end{array}$ & $\begin{array}{l}18.6 \\
(6.90)\end{array}$ & $\begin{array}{l}18.1 \\
(6.58)\end{array}$ & $\begin{array}{l}18.2 \\
(7.65)\end{array}$ & $\begin{array}{l}18.0 \\
(6.95)\end{array}$ & 0.542 \\
\hline \multicolumn{7}{|l|}{ Bicycle lane (\%) } \\
\hline $\begin{array}{l}\text { Home, proportion in } \\
400 \mathrm{~m} \text { buffer }\end{array}$ & 13.8 & 15.6 & 15.4 & 13.2 & 11.2 & 0.002 \\
\hline $\begin{array}{l}\text { Work/study,proportion in } \\
400 \mathrm{~m} \text { buffer }\end{array}$ & 29.7 & 31.9 & 33.5 & 28.7 & 25.5 & 0.009 \\
\hline $\begin{array}{l}\text { Commute } \\
\text { route,proportion in } R B A\end{array}$ & 13.7 & 14.8 & 14.2 & 13.8 & 12.0 & $<0.001$ \\
\hline $\mathrm{NO}_{2}, \mathrm{ppb}[\operatorname{mean}(\mathrm{SD})]$ & & & & & & \\
\hline
\end{tabular}




\begin{tabular}{|c|c|c|c|c|c|c|}
\hline Descriptive & $\begin{array}{l}\text { All } \\
n=769\end{array}$ & $\begin{array}{l}\text { Bicyclist, } \\
\text { Frequent } \\
n=281\end{array}$ & $\begin{array}{l}\text { Bicyclist, } \\
\text { Infrequent } \\
n=120\end{array}$ & $\begin{array}{l}\text { Non- } \\
\text { bicyclist, } \\
\text { Willing } \\
n=141\end{array}$ & $\begin{array}{l}\text { Non- } \\
\text { bicyclist, } \\
\text { Unwilling } \\
n=227\end{array}$ & $\begin{array}{l}p- \\
\text { value }{ }^{a}\end{array}$ \\
\hline $\begin{array}{l}\text { Home, concentration in } \\
400 \mathrm{~m} \text { buffer }\end{array}$ & $\begin{array}{l}76.2 \\
(17.5)\end{array}$ & $\begin{array}{l}78.1 \\
(17.6)\end{array}$ & $\begin{array}{l}74.9 \\
(18.4)\end{array}$ & $\begin{array}{l}74.6 \\
(17.2)\end{array}$ & $\begin{array}{l}75.6 \\
(17.1)\end{array}$ & 0.091 \\
\hline $\begin{array}{l}\text { Work/study, concentration } \\
\text { in } 400 \text { m buffer }\end{array}$ & $\begin{array}{l}78.5 \\
(22.5)\end{array}$ & $\begin{array}{l}79.2 \\
(21.1)\end{array}$ & $\begin{array}{l}77.3 \\
(21.4)\end{array}$ & $\begin{array}{l}77.6 \\
(24.1)\end{array}$ & $\begin{array}{l}78.7 \\
(23.9)\end{array}$ & .738 \\
\hline $\begin{array}{l}\text { Commute } \\
\text { route,concentration in } \\
R B A\end{array}$ & $\begin{array}{l}84.4 \\
(17.0)\end{array}$ & $\begin{array}{l}85.2 \\
(17.4)\end{array}$ & $\begin{array}{l}83.5 \\
(16.2)\end{array}$ & $\begin{array}{l}82.3 \\
(16.0)\end{array}$ & $\begin{array}{l}85.3 \\
(17.3)\end{array}$ & 0.257 \\
\hline \multicolumn{7}{|l|}{ Noise, >55 dB (\%) } \\
\hline $\begin{array}{l}\text { Home, proportion in } \\
400 \mathrm{~m} \text { buffer }\end{array}$ & 78.5 & 78.5 & 78.6 & 77.8 & 78.9 & 0.682 \\
\hline $\begin{array}{l}\text { Work/study,proportion in } \\
400 \mathrm{~m} \text { buffer }\end{array}$ & 79.6 & 79.4 & 81.3 & 80.5 & 78.3 & 0.396 \\
\hline $\begin{array}{l}\text { Commute } \\
\text { route,proportion in RBA }\end{array}$ & 77.3 & 76.7 & 78.5 & 78.2 & 77.0 & 0.160 \\
\hline \multicolumn{7}{|l|}{$\begin{array}{l}\text { Greenness, NDVI [IQR, } \\
\text { mean(SD)] }\end{array}$} \\
\hline $\begin{array}{l}\text { Home, average of } 400 \mathrm{~m} \\
\text { buffer }\end{array}$ & $\begin{array}{l}0.79 \\
(1.07)\end{array}$ & $\begin{array}{l}0.61 \\
(0.95)\end{array}$ & $\begin{array}{l}0.86 \\
(1.27)\end{array}$ & $\begin{array}{l}0.91 \\
(1.16)\end{array}$ & $\begin{array}{l}0.90 \\
(1.02)\end{array}$ & 0.002 \\
\hline $\begin{array}{l}\text { Work/study, average of } \\
400 \mathrm{~m} \text { buffer }\end{array}$ & $\begin{array}{l}0.62 \\
(0.96)\end{array}$ & $\begin{array}{l}0.52 \\
(0.83)\end{array}$ & $\begin{array}{l}0.60 \\
(0.82)\end{array}$ & $\begin{array}{l}0.77 \\
(1.03)\end{array}$ & $\begin{array}{l}0.68 \\
(1.11)\end{array}$ & 0.177 \\
\hline $\begin{array}{l}\text { Commute route, average } \\
\text { of } R B A\end{array}$ & $\begin{array}{l}0.97 \\
(0.96)\end{array}$ & $\begin{array}{l}0.84 \\
(0.82)\end{array}$ & $\begin{array}{l}0.94 \\
(0.91)\end{array}$ & $\begin{array}{l}1.03 \\
(1.00)\end{array}$ & $\begin{array}{l}1.10 \\
(1.11)\end{array}$ & 0.212 \\
\hline \multicolumn{7}{|l|}{ Elevation [mean(SD)] } \\
\hline $\begin{array}{l}\text { Home, average of } 400 \mathrm{~m} \\
\text { buffer }\end{array}$ & $\begin{array}{l}42.7 \\
(49.0)\end{array}$ & $\begin{array}{l}35.4 \\
(30.7)\end{array}$ & $\begin{array}{l}37.5 \\
(39.7)\end{array}$ & $\begin{array}{l}45.4 \\
(40.9)\end{array}$ & $\begin{array}{l}52.8 \\
(44.1)\end{array}$ & $<0.001$ \\
\hline $\begin{array}{l}\text { Work/study, average of } \\
400 \mathrm{~m} \text { buffer }\end{array}$ & $\begin{array}{l}40.6 \\
(40.0)\end{array}$ & $\begin{array}{l}31.6 \\
(26.2)\end{array}$ & $\begin{array}{l}37.5 \\
(33.0)\end{array}$ & $\begin{array}{l}43.5 \\
(43.0)\end{array}$ & $\begin{array}{l}51.6 \\
(51.4)\end{array}$ & $<0.001$ \\
\hline
\end{tabular}

a ANOVA or Kruskal-wallis for normally and abnormally-distributed continuous variables, respectively. 
Adults ( $n=769 ; 52 \%$ females) commuting to work or school in Barcelona responded to a comprehensive telephone survey concerning their travel behaviour, through June 2011 to May 2012. Based upon responses, participants were categorised into four groups of propensity to bicycle commute: frequent bicyclists, infrequent bicyclists, willing non-bicyclists, and unwilling non-bicyclists. Environmental (GIS) determinants were calculated using ArcGIS and a participant's geocoded home and work/study address, as either 400 metre buffers surrounding these addresses or a route-by-area (RBA) surrounding the predicted route and the two addresses. 


\section{Adjusted models of environmental determinants}

Personal determinants of age, education level, gender, nationality, vigorous exercise performance and commute distance were included in the final multinomial logistic regression model. Testing statistically for model goodness of fit, the final model has a Prob > chi-squared of 0.327 .

Nationality and vigorous exercise were significant positive determinants of being an infrequent bicycle commuter rather than an unwilling nonbicycle commuter. On the other hand, gender and commute distance were a significant negative determinant of this. Nationality was a significant positive determinant of being a frequent bicycle commuter rather than an unwilling non-bicycle commuter, while age, gender and commute distance were significant negative determinants of this.

Similarly, nationality was a positive, while education and gender were negative, significant determinants of being a willing rather than an unwilling non-bicycle commuter (Table 3 ). 
Table 3.

Adjusted multinomial regression model with environmental and personal determinants of bicycle commuting; according to individual propensity for bicycle commuting.

\section{Determinant}

ENVIRONMENTAL

Public bicycle stations

Home, count in $400 m$ buffer

Public transport stations

Home, count in 400m buffer

Greenness, NDVI

Work/study, IQR (mean) of $400 \mathrm{~m}$

buffer

Elevation

Work/study, mean of $400 m$ buffer

\section{PERSONAL}

Age, continuous

Education, dichotomous,

tertiary-educated

Gender, dichotomous,

Female

Nationality, dichotomous,

Spanish

Vigorous exercise (weekly),

dichotomous, Affirmative

Commute distance (trip),

continuous

Abbreviation, definition: RRR, relative risk ratio; 95\%CI, 95\% confidence interval; NDVI, Normalized Difference Vegetation Index; IQR, inter-quartile range.

\begin{tabular}{|c|c|c|}
\hline $\begin{array}{l}\text { Non-bicyclists, } \\
\text { Unwilling: } \\
\text { Bicyclists, Frequent }\end{array}$ & $\begin{array}{l}\text { Non-bicyclists, } \\
\text { Unwilling: } \\
\text { Bicyclists, Infrequent }\end{array}$ & $\begin{array}{l}\text { Non-bicyclists, Unwilling } \\
\text { Non-bicyclists, Willing }\end{array}$ \\
\hline $\operatorname{RRR}(95 \% \mathrm{Cl})$ & $\operatorname{RRR}(95 \% \mathrm{Cl})$ & $\operatorname{RRR}(95 \% \mathrm{Cl})$ \\
\hline $1.121(1.032-1.216)$ & 1.105 (1.004-1.216) & $0.969(0.883-1.062)$ \\
\hline $0.930(0.895-0.966)$ & 0.955 (0.913-0.999) & $0.973(0.935-1.014)$ \\
\hline 1.537 (1.136-2.079) & $1.435(1.009-2.042)$ & $1.693(1.221-2.347)$ \\
\hline $0.978(0.971-0.986)$ & $0.985(0.976-0.994)$ & $0.987(0.980-0.995)$ \\
\hline $0.971(0.952-0.991)$ & $0.980(0.957-1.003)$ & $0.999(0.979-1.021)$ \\
\hline $1.480(0.933-2.349)$ & $1.005(0.585-1.725)$ & $0.442(0.279-0.701)$ \\
\hline $0.371(0.248-0.556)$ & $0.397(0.245-0.644)$ & $0.515(0.327-0.809)$ \\
\hline $2.581(1.317-5.059)$ & 3.844 (1.867-7.916) & $2.587(1.265-5.288)$ \\
\hline $1.467(0.976-2.206)$ & $2.247(1.342-3.761)$ & $0.845(0.537-1.329)$ \\
\hline $0.690(0.618-0.771)$ & $0.831(0.735-0.939)$ & $0.931(0.841-1.030)$ \\
\hline
\end{tabular}


Adults ( $n=769 ; 52 \%$ females) commuting to work or school in Barcelona responded to a comprehensive telephone survey concerning their travel behaviour, through June 2011 to May 2012. Based upon responses, participants were categorised into four groups of propensity to bicycle commute: frequent bicyclists, infrequent bicyclists, willing non-bicyclists, and unwilling non-bicyclists. The determinants of frequency and willingness (propensity) to commute by bicycle were assessed by a multinomial logistic regression model adjusted for personal features (potential confounders and covariates) including age, education level, gender, nationality, physical activity and commute distance. Unwilling non-bicyclists were used as the base for comparison in this model to indicate the increased likelihood of bicycling or being willing to bicycle. Environmental (GIS) determinants were calculated using ArcGIS and a participant's geocoded home and work/study address, as either 400 metre buffers surrounding these addresses or a route-by-area (RBA) surrounding the predicted route between the two addresses. 
The environmental determinants positively-determining being a bicycle commuter (either frequent or infrequent) rather than an unwilling nonbicycle commuter were the quantity of public bicycle (Bicing) stations within the home area and greenness within the work/study area. However, the quantity of public transport stations within the home area and the mean elevation of the work/study area were both negativelydetermining this (Table 3 ).

The amount of greenness within the work/study area was a positive determinant of being a willing non-bicycle commuter rather than an unwilling non-bicycle commuter. The same, but negative, determination was observed for the mean elevation within the work/study area (Table 3).

Sensitivity analysis showed that population density did not remove significance from model outcomes (results not shown). Supplemental analysis stratifying the model by occupation status (worker/student) showed that some outcomes lost significance for students (Appendix Table A). Our study sample was observed to be representative of the general population surveyed in the Barcelona Active Population Survey (BAPS), in terms of age, gender and neighbourhood socioeconomic status (Agència de Salut Pública de Barcelona, 2011) (Appendix Figs. $\mathrm{C}$ and $\mathrm{D})$.

\section{Discussion}

The current study aimed to identify which urban environmental determinants affect the propensity to commute by bicycle within a city such as Barcelona, Spain. In the adjusted multinomial logistic regression model, significant positive determinants of propensity for bicycle commuting were the quantity of public bicycle stations within the home area, and amount of greenness within the work/study area (i.e. within a $400 \mathrm{~m}$ radius). On the other hand, the quantity of public transport stations within the home area and the mean elevation of the work/study area were significant negative determinants of the same propensity. Personal characteristics such as age, education level, gender, nationality, vigorous exercise and commute distance were all associated with this propensity. While some of these observations support previous findings (such as infrastructure, topography and personal characteristics), the suggestion that the amount of objectivelymeasured greenness and public bicycle sharing stations have a positive effect on bicycle commuting propensity is novel. Moreover, while many mode choice analyses have examined objective measures of bicycling infrastructure, most have considered these in proximity to 
home addresses and relatively few have examined infrastructure characteristics within the work/study environment, where in the current study greenness was seen to be a positive determinant for bicycle commuting.

The number of public bicycle (Bicing) stations within the home, although not the work/study area, was a significant positive determinant of being a bicycle commuter rather than a non-bicycle commuter. This suggests that availability of public bicycle stations may motivate nonbicycle commuters to begin bicycle commuting. It could be argued that stations were deployed to areas indicated by expected use, or that individuals already prone to bicycle commuting were drawn to reside in areas with such infrastructure, but the Bicing stations have been deployed evenly throughout the city (at approximately even distances apart). Further, the fact that our finding was robust with sensitivity analysis using population density supports against these previous arguments. Public bicycle sharing schemes are relatively new infrastructure and therefore less studied than bicycle parking for private bikes; however, it has been seen previously that bicycle parking provision increases the odds for bicycle commuting, even when controlling for other explanatory variables (Buehler, 2012). Arguably, such parking increases the convenience of bicycle use for commuting, which could be conveyed with public bicycle stations available at the home address area. Other reasons for the presence of Bicing stations positively influencing bicycle commuting propensity may include the higher visibility of people using bicycles for commuting (i.e. arriving/departing to/from the station).

Besides convenience and observation, another source of motivation for non-bicyclists to use public bicycle sharing schemes may come from the flexibility associated with such systems, allowing one-way commute trips by bicycle from the home (origin) to work/study (destination) address. Such flexibility may be particularly attractive to users that have a destination at a higher elevation than the origin, requiring extra physical effort to gain altitude, even if the user is physically capable of the effort (reflected by our study population). We observed that the mean elevation of the work/study area was a negative determinant for non-bicyclists to be willing to bicycle commute. Similar observations have been made extensively before (Broach et al., 2012, Rodríguez and Joo, 2004, Troped et al., 2001, Hood et al., 2011 and Heinen et al., 2012). Unfortunately, topography is difficult to address with policy, although measures such as electrically-assisted bicycles, now being introducing by Bicing in Barcelona, may help to address this apparent deterrent. Another possibility is to have bicycle sharing systems provide 
monetary incentives (for example a discounted ride) for users that park the bicycle in locations with higher elevation relative to other locations in the system. Moreover, having public bicycle stations within the home area may be particularly important in cities like Barcelona for nonbicyclists due to relatively-low bicycle access (at approximately 40$60 \%$ of our non-bicyclist respondents) compared to exceptional cities such as Copenhagen (at approximately $80 \%$ of residents) (City of Copenhagen 2013).

The number of public transport stations within the home area was negatively-associated with bicycle commuting participation. It may be reasonable then to suggest that public transport use is competing with bicycle use for commuting (depending on infrastructure availability); however, it has been previously shown in Spain that the proximity of public transport to commute addresses is not significantly negativelycorrelated with participation in active transport (Molina-García et al., 2010).

Interestingly, we observed that the greenness surrounding a work/study address was a significant positive determinant of bicycle commuting. To the authors' knowledge this association with objective (NDVI) measures is novel. Green space, when incorporated into the plan of an urban environment, has been previously suggested as a method to increase physical activity participation (Lee and Maheswaran, 2011) and has been reported as the most stimulating perceived (subjective) environmental determinant of bicycle commuting (Wahlgren and Schantz, 2012). It may be thought that lower-density areas, of lower traffic, are greener; however, when testing for sensitivity to population density of the work/study address our finding was robust. In Barcelona and many other major cities, motorised traffic is the major emitter of air pollution and noise, making it difficult to determine if it is the lack of traffic (and thus emissions) or the presence of greenness (or a mixture of both) determining propensity for bicycle commuting. It has been previously observed that a strong (self-reported) motivator for the decision to bicycle commute or not by both bicyclists and non-bicyclists are routes with appealing scenery (Winters et al., 2011), however we did not observe greenness of the (estimated) commute route to be significantly determining bicycle commuting propensity. The possible issue of collinearity of greenness with bicycle lanes (being significantly correlated with bicycle commuting propensity although not a significant determinant within the multinomial model) was ruled-out by (1) insignificant correlations with bivariate analysis of final model independent variables, and (2) negligible effect on model fit statistics when replacing greenness level with bicycle lane proportion at the 
work/study address (results not shown). Further, the fact that greenness of only the work/study address was significantly determining bicycle commuting propensity may reveal that other characteristics of the work/study location or profession type (such as industrial zoning, irregular work hours, carried equipment, physical labour, etcetera) associated with greenness may be the true determinants. Besides environmental determinants, we found age, education level, gender, nationality, vigorous exercise and commute distance to be important for consideration of demographics and their influence on attitudes and behavioural control (propensity to bicycle commute), in agreement with previous studies (Dill et al., 2014, Sener et al., 2009a, Akar and Clifton, 2009 and Parkin et al., 2007). When stratifying the analysis by occupation status as a potential effect modifier in the association between determinants of interest and the behaviours, the result was that outcomes lost some significance within the student subsample. This was probably due to the small sub-sample size of students and therefore statistical power. While more interesting results may have been expected from such stratification by occupation status (Heinen et al., 2010), it should be noted that our sample were all adults. Additionally, there was not a significant difference in proportion of students between propensity groups. Moreover, while respondents with very short commute distances were excluded from this study (as they are assumed to walk), there was no maximum distance considered for exclusion. This is because respondents had to have a home and work/study address within Barcelona, which is a relatively small city in area (approximately $4 \mathrm{~km}$ radius), therefore commute distances were considered within the limits of bicycle use.

Although our results from the adjusted models show few significant environmental correlates associated with the bicycling outcomes, it is important to note that all measured correlates were included in the model. Many of the exposures are likely to be correlated. Although we tested sensitivity to population density in our model, arguably, busier parts of the city are likely to have a higher public transport service and quantity of Bicing stations. Average variance inflation factors were 3 , but some were as high as 6 . This means that the broad built and natural environments, as measured here, are likely to be associated with the outcomes. Identifying the specific characteristics of those environments is much more challenging and to be addressed in future research. Despite an extensive recruitment drive, a relatively low response rate was achieved. Less than half of those individuals approached for recruitment completed the survey, potentially adding bias to the response data set due to respondent motivation. Likely bias would 
nevertheless tend to over-represent individuals with a more active lifestyle and therefore underestimate real differences due to the built environment. While the study sampling was spatiotemporallyrandomised throughout the city, which enjoys a Mediterranean climate with relatively stable meteorological conditions, the recruitment period was long and therefore response bias may come from seasonal variation in bicycling levels. Further, GIS environmental data was attributed to a respondent according to the estimation of a 'shortest route' and the application of a 'route-by-area' between home and work/study address. This attribution may be inaccurate as bicyclists have been shown to travel longer distances to use bicycle infrastructure such as bicycle lanes (Dill and Gliebe, 2008). Therefore, our findings may not be completely transferable to the general population. While our study population was reflective of the general city demographic (see Appendix Figs. C and D), some small differences existed in that our study population slightly over-represented the first and second quartile of neighbourhood socioeconomic index, under 40 year olds, and women. We did not collect participant occupation type and therefore could not know if greenness at the work/study address is the determinant of effect, or a confounder of occupation characteristics, on bicycle commuting propensity. Additionally, the estimation of commute route did not allow for adjustment of our models with (known) commute distance, only that calculated within a GIS environment. Furthermore, our analyses did not directly address the issue of perceptions as a determinant in travel decisions, which is considered important and may differ from objective measures (Dill and Voros, 2007, Hoehner et al., 2005 and McGinn et al., 2007) - future studies may attempt to consider both to determine which is more important for policy measures. Also, future studies should ask for occupation type to determine if the occupation description is confounding with variables of the work address environment. Future research may also use GPS-measured commute routes for better environmental determinant attribution, such as by using a uniform buffer along the commute route to better capture variables such as bicycle lanes, rather than relying on GIS networking and a route-byarea approach.

To our knowledge, this is the first study providing information on objective measures of an urban environment in a Mediterranean city most studies are from northern Europe or the USA (Heinen et al., 2010). Additionally, this is the first study to consider the influence of objectively-measured greenness on an individual's propensity to bicycle commute, suggesting that it (or related factor/s) may be a 
positive determinant of bicycle commuting. In general, an integral strength of the current study is the use of a comprehensive GIS dataset. Another strength is that the current weekly frequency of bicycle commuting is included as a dependent variable to further define (and validate) the different sub-groups of self-reported bicycle commuting propensity. Further, we considered the willingness to start bicycle commuting within non-bicycle commuters, which we could contribute to consideration of behaviour change interventions on nonusers. We have been able to compare the influence of environmental and personal determinants between bicycle commuters and nonbicycle commuters of different propensities. This is a novel and reasonable approach to analysing survey response data, allowing sufficient statistical strength for comparing groups according to bicycle commuting propensity and identifying the significant determinants of this.

\section{Conclusion}

Our findings suggest that the presence of greenness and moreso public bicycle stations are significant positive determinants for an individual's propensity to bicycle commute. Alternatively, the negative association suggests public transit competes with bicycling commuting. Elevation change between the trip origin and destination deters bicycle commuting as expected. These determinants should be further considered with future research and transport policy on bicycle commuting; doing so would support an increase in city commuting by bicycle and consequently help to address physical inactivity, vehicular congestion, air pollution and climate change.

\section{Acknowledgements}

We would like to sincerely thank Tania Martínez, Jaume Matamala, and Meritxell Portella for assistance with participant recruitment and data collection. We would like to acknowledge the ESCAPE project and its contributors for air quality model data of Barcelona, as well as the Ajuntament de Barcelona and the Generalitat de Catalunya for noise model data and street map information. This study was performed as part of the TAPAS project <http://www.tapas-program.org/>, funded by the Coca-Cola Foundation and the Agència de Gestió d'ajuts Universitaris i de Recerca (AGAUR).

\section{Appendix A. Supplementary material}


Participant recruitment points: (a) by district; and (b) home addresses in relation to population density. To reach a variety of commuters in the street, 40 recruitment points covered the expanse of Barcelona city, comprising four randomly-selected points in each of the ten city districts. Within proximity of each point, strategic locations were chosen (when available) to target public transport users near public transport stations, bicycle commuters near Bicing stations, and private transport users near vehicle parking lots. Each point was sampled by three trained interviewers between 7:45 and 11:30 a.m. during four days within a randomly selected week.

Supplementary Appendix Fig. B.

Spatial distribution and linear relationship between participants' home address and (a) population density of Barcelona or (b) commercial outlet density of Barcelona.

Supplementary Appendix Fig. C.

Comparison of study sample age and gender distribution against the Barcelona Active Population Survey (BAPS).

Supplementary Appendix Fig. D.

Comparison of study sample home neighbourhood deprivation index distribution against the Barcelona Active Population Survey (BAPS).

Supplementary Appendix Table A.

Adjusted multinomial regression model with environmental and personal determinants of bicycle commuting; according to individual propensity for bicycle commuting, according to worker or student status.

\section{References}

Agència de Salut Pública de Barcelona, 2011

Agència de Salut Pública de Barcelona. La Salut a Barcelona 2011. Barcelona: 2012. <http://www.aspb.cat/quefem/docs/Informe Salut 2011.pdf>.

Ajuntament de Barcelona, 2010 Ajuntament de Barcelona. Pla d'acció per a la minoració de la contaminació acústica de la ciutat de Barcelona. 2010. http://sima.gencat.cat/Visors/Soroll//nici.html.

Ajuntament de Barcelona, 2011 Ajuntament de Barcelona. Public map of private bicycle parking. 2011.

$<$ http://w3.bcn.cat/fitxers/mobilitat/bici/20100227reservesbicicletesokversio.417. pdf $>$. 
Akar and Clifton, 2009 G. Akar, K.J. Clifton Influence of individual perceptions and bicycle infrastructure on decision to bike Transp. Res. Rec. J. Transp. Res. Board, 2140 (2009), pp. 165172 http://dx.doi.org/10.3141/2140-18

Autoritat del Transport Metropolità, 2013 Autoritat del Transport Metropolità. Enquesta de mobilitat en dia feiner. Barcelona: 2013.

$<$ <ttp://www.iermb.uab.es/htm/mobilitat/pdf/resultatsEMEF2012.pdf>.

Beyer, 2012 Beyer, H.L., 2012. Geospatial Modelling Environment. $<$ http://www.spatialecology.com/gme>.

Beelen et al., 2013 R. Beelen, G. Hoek, D. Vienneau, et al. Development of $\mathrm{NO}_{2}$ and $\mathrm{NOx}$ land use regression models for estimating air pollution exposure in $\mathbf{3 6}$ study areas in Europe - The ESCAPE project Atmos. Environ., 72 (2013), pp. 10-23 http://dx.doi.org/10.1016/..atmosenv.2013.02.037

Bhatia and Wier, 2011 R. Bhatia, M. Wier "Safety in Numbers" re-examined: can we make valid or practical inferences from available evidence? Accid. Anal. Prev., 43 (2011), pp. 235-240 http://dx.doi.org/10.1016/j.aap.2010.08.015

Boarnet and Crane, 2001 M. Boarnet, R. Crane The influence of land use on travel behavior: specification and estimation strategies Transp. Res. Part A Policy Pract., 35 (2001)

<http://www.sciencedirect.com/science/article/pii/S0965856400000197> (accessed 21.04.15)

Broach et al., 2012 J. Broach, J. Dill, J. Gliebe Where do cyclists ride? A route choice model developed with revealed preference GPS data Transp. Res. Part A Policy Pract., 46 (2012), pp. 17301740http://dx.doi.org/10.1016/j.tra.2012.07.005

Brown et al., 2013 B. Brown, K. Smith, H. Hanson Neighborhood design for walking and biking: physical activity and body mass index Am. J. Prev. Med., 44 (2013), pp. 231-238 <http://www.sciencedirect.com/science/article/pii/S074937971200880X> (accessed 29.11.13)

Buehler, 2012 R. Buehler Determinants of bicycle commuting in the Washington, DC region: The role of bicycle parking, cyclist showers, and free car parking at work Transp. Res. Part D Transp. Environ., 17 (2012), pp. 525-531 http://dx.doi.org/10.1016/j.trd.2012.06.003 


\section{Cervero, 2002}

R. Cervero Built environments and mode choice: toward a normative framework Transp. Res. Part D Transp. Environ., 7 (2002), pp. 265284 http://dx.doi.org/10.1016/S1361-9209(01)00024-4

\section{City of Copenhagen, 2012}

City of Copenhagen. Bicycle Account 2012. Copenhagen: 2013.

<http://kk.sites.itera.dk/apps/kk pub2/pdf/1034 pN9YE5rO1u.pdf>.

Craig et al., 2003 C.L. Craig, A.L. Marshall, M. Sjöström, et al. International physical activity questionnaire: 12-country reliability and validity Med. Sci. Sports Exerc., 35 (2003), pp. 1381-1395 http://dx.doi.org/10.1249/01.MSS.0000078924.61453.FB

Crane, 1996 R. Crane On form versus function: will the new urbanism reduce traffic, or increase it? J. Plan. Educat. Res. (1996), pp. 117-126 $<$ http://ipe.sagepub.com/content/15/2/117.short> (accessed 21.04.15)

De Nazelle et al., 2011 A. De Nazelle, M.J. Nieuwenhuijsen, J.M. Antó, et al. Improving health through policies that promote active travel: a review of evidence to support integrated health impact assessment Environ. Int., 37 (2011), pp. 766-777 http://dx.doi.org/10.1016/j.envint.2011.02.003

Departament d'Estadística, 2011 Departament d'Estadística. INFORMES ESTADÍSTICS Cens de vehicles de la ciutat de Barcelona. Barcelona: 2011. $<$ <ttp://www.bcn.cat/estadistica/catala/dades/inf/veh/veh11/veh11.pdf>.

Desarrollo Organización Movilidad, 2011 Desarrollo Organización Movilidad. Dades bàsiques de mobilitat 2011. Barcelona: 2012. $<$ http://w110.bcn.cat/Mobilitat/Continguts/Documents/Fitxers/dadesbasiques201 1 complert.pdf $>$.

Dill and Gliebe, 2008 Dill, J., Gliebe, J., 2008. Understanding and measuring bicycling behavior: a focus on travel time and route choice.

<http://dr.archives.pdx.edu/xmlui/handle/psu/7940>.

Dill and Voros, 2007

J. Dill, K. Voros Factors affecting bicycling demand: initial survey findings from the Portland, Oregon, Region Transp. Res. Rec., 2031 (2007), pp. 917 http://dx.doi.org/10.3141/2031-02 
Dill et al., 2014 J. Dill, C. Mohr, L. Ma How can psychological theory help cities increase walking and bicycling? J. Am. Plan. Assoc., 80 (2014), pp. 36-51 http://dx.doi.org/10.1080/01944363.2014.934651

Ewing et al., 2008 R. Ewing, K. Bartholomew, S. Winkelman, et al. Urban development and climate change J. Urban Int. Res. Placemaking Urban Sustain., 1 (2008), pp. 116-201

$<$ http://www.tandfonline.com/doi/abs/10.1080/17549170802529316> (accessed 23.04.15)

Federació de Municipis de Catalunya, 2008 Federació de Municipis de Catalunya, Barcelona (Catalunya). Ajuntament, Institut d'Estudis Regionals i Metropolitans de Barcelona, et al. La Mobilitat quotidiana a Catalunya. Barcelona:: Institut d'Estudis Regionals i Metropolitans de Barcelona 2008.

Forsyth and Krizek, 2012 A. Forsyth, K. Krizek Reliability testing of the Pedestrian and Bicycling Survey (PABS) method J. Phys. Act. Health, 9 (2012), pp. 677-688<http://kevinjkrizek.org/wpcontent/uploads/2012/04/ForsythKrizekAgrawalStonebreaker 2012 PABS1.pdf $>$ (accessed 29.11.13)

Frank et al., 2007 L. Frank, J. Kerr, J. Chapman, et al. Urban form relationships with walk trip frequency and distance among youth Am. J. Health Promot., 21 (2007), pp. 305-311 http://dx.doi.org/10.4278/0890-1171$\underline{21.4 s .305}$

Furie and Desai, 2012 G.L. Furie, M.M. Desai Active transportation and cardiovascular disease risk factors in U.S. adults Am. J. Prev. Med., 43 (2012), pp. 621-628 http://dx.doi.org/10.1016/i.amepre.2012.06.034

Garrard et al., 2008 J. Garrard, G. Rose, S.K. Lo Promoting transportation cycling for women: the role of bicycle infrastructure Prev. Med. (Baltim), 46 (2008), pp. 55-59 http://dx.doi.org/10.1016/j.ypmed.2007.07.010

Generalitat de Catalunya, 2011 Generalitat de Catalunya. Transports. 2011. $<$ http://www20.gencat.cat/portal/site/territori/menuitem.c6e8d3be598ec9745f13a e92b0c0e1a0/?vgnextoid=f35915077e456310VgnVCM1000008d0c1e0aRCRD\& vgnextchannel=f35915077e456310VgnVCM1000008d0c1e0aRCRD\&vgnextfmt =default>. 
Handy and Boarnet, 2002 S. Handy, M. Boarnet How the built environment affects physical activity: views from urban planning Am. J. Prev. Med., 23 (2002) <http://www.sciencedirect.com/science/article/pii/S0749379702004750> (accessed 23.04.15)

Haskell et al., 2007 W.L. Haskell, I.-M. Lee, R.R. Pate, et al. Physical activity and public health: updated recommendation for adults from the American College of Sports Medicine and the American Heart Association Med. Sci. Sports Exerc., 39 (2007), pp. 14231434 http://dx.doi.org/10.1249/mss.0b013e3180616b27

Heinen et al., 2010 E. Heinen, B. van Wee, K. Maat Commuting by bicycle: an overview of the literature Transp. Rev., 30 (2010), pp. 5996 http://dx.doi.org/10.1080/01441640903187001

Heinen et al., 2012 E. Heinen, K. Maat, B. Wee The effect of work-related factors on the bicycle commute mode choice in the Netherlands Transportation (Amst), 40 (2012), pp. 23-43 http://dx.doi.org/10.1007/s11116$\underline{012-9399-4}$

Hoehner et al., 2005 C.M. Hoehner, L.K. Brennan Ramirez, M.B. Elliott, et al. Perceived and objective environmental measures and physical activity among urban adults Am. J. Prev. Med., 28 (2005), pp. 105116 http://dx.doi.org/10.1016/j.amepre.2004.10.023

Hood et al., 2011 J. Hood, E. Sall, B. Charlton A GPS-based bicycle route choice model for San Francisco, California Transp. Lett. Int. J. Transp. Res., 3 (2011), pp. 63-75 http://dx.doi.org/10.3328/TL.2011.03.01.63-75

James et al., 2015 P. James, R.F. Banay, J.E. Hart, et al. A review of the health benefits of greenness Curr. Epidemiol. Reports (2015) http://dx.doi.org/10.1007/s40471-015-0043-7

Lee and Maheswaran, 2011 A.C.K. Lee, R. Maheswaran The health benefits of urban green spaces: a review of the evidence J. Public Health (Oxf), 33 (2011), pp. 212-222 http://dx.doi.org/10.1093/pubmed/fdq068

Lee et al., 1999 I.-M. Lee, C.H. Hennekens, K. Berger, et al. Exercise and risk of stroke in male physicians Stroke, 30 (1999), pp. 16 http://dx.doi.org/10.1161/01.STR.30.1.1 
Lim et al., 2012 S.S. Lim, T. Vos, A.D. Flaxman, et al.A comparative risk assessment of burden of disease and injury attributable to 67 risk factors and risk factor clusters in 21 regions, 1990-2010: a systematic analysis for the Global Burden of Disease Study 2010 Lancet, 380 (2012), pp. 22242260 http://dx.doi.org/10.1016/S0140-6736(12)61766-8

McGinn et al., 2007 A.P. McGinn, K.R. Evenson, A.H. Herring, et al.Exploring associations between physical activity and perceived and objective measures of the built environment J. Urban Health Bull. New York Acad. Med., 84 (2007), pp. 162-184 http://dx.doi.org/10.1007/s11524-006-9136-4

Midgley, 2011 Midgley P. Bicycle-sharing schemes: enhancing sustainable mobility in urban areas. New York, New York, USA: 2011.

$<$ http://www.un.org/esa/dsd/resources/res_pdfs/csd-19/Background-Paper8-

P.Midgley-Bicycle.pdf> (accessed 24.02.15).

Ministere de L'Environnement, 1980 Ministere de L'Environnement. Prevision des Niveaux Sonores. 1980. <http://temis.documentation.developpementdurable.gouv.fr/documents/temis/4859/4859_1980.pdf>.

Molina-García et al., 2010 J. Molina-García, I. Castillo, J.F. Sallis Psychosocial and environmental correlates of active commuting for university students Prev. Med. (Baltim), 51 (2010), pp. 136138 http://dx.doi.org/10.1016/j.ypmed.2010.05.009

Ortúzar et al., 2000 J.D.D. Ortúzar, A. lacobelli, C. Valeze Estimating demand for a cycle-way network Transp. Res. Part A Policy Pract., 34 (2000), pp. 353 373 http://dx.doi.org/10.1016/S0965-8564(99)00040-3

Panter and Jones, 2010 J.R. Panter, A. Jones Attitudes and the environment as determinants of active travel in adults: what do and don't we know? J. Phys. Act Health, 7 (2010), pp. 551-561 <http://www.ncbi.nlm.nih.gov/pubmed/20683098

Panter et al., 2008 J.R. Panter, A.P. Jones, E.M. van Sluijs Environmental determinants of active travel in youth: a review and framework for future research Int. J. Behav. Nutr. Phys. Act., 5 (2008), p. 34 http://dx.doi.org/10.1186/1479-5868-5-34

Parkin et al., 2007 J. Parkin, M. Wardman, M. Page Estimation of the determinants of bicycle mode share for the journey to work using census 
data Transportation (Amst), 35 (2007), pp. 93-

109 http://dx.doi.org/10.1007/s11116-007-9137-5

Pucher et al., 2010 J. Pucher, J. Dill, S. Handy Infrastructure, programs, and policies to increase bicycling: an international review Prev. Med. (Baltim), 50 (Suppl 1) (2010), pp. S106-

S125http://dx.doi.org/10.1016/j.ypmed.2009.07.028

Pucher et al., 2010 J. Pucher, R. Buehler, D.R. Bassett, et al. Walking and cycling to health: a comparative analysis of city, state, and international data Am. J. Public Health, 100 (2010), pp. 19861992 http://dx.doi.org/10.2105/AJPH.2009.189324

Rodríguez and Joo, 2004 D.A. Rodríguez, J. Joo The relationship between non-motorized mode choice and the local physical environment Transp. Res. Part D Transp. Environ., 9 (2004), pp. 151173http://dx.doi.org/10.1016/j.trd.2003.11.001

Rodriguez et al., 2014 D.A. Rodriguez, L. Merlin, C.G. Prato, et al. Influence of the Built Environment on Pedestrian Route Choices of Adolescent Girls Environ Behav, 47 (2014), pp. 359394 http://dx.doi.org/10.1177/0013916513520004

Sener et al., 2009a I.N. Sener, N. Eluru, C.R. Bhat Who are bicyclists? Why and how much are they bicycling? Transp. Res. Rec. J. Transp. Res. Board, 2134 (2009), pp. 63-72 http://dx.doi.org/10.3141/2134-08

Sener et al., 2009b I.N. Sener, N. Eluru, C.R. Bhat An analysis of bicycle route choice preferences in Texas, US Transportation (Amst), 36 (2009), pp. 511539 http://dx.doi.org/10.1007/s11116-009-9201-4

Titze et al., 2008 S. Titze, W.J. Stronegger, S. Janschitz, et al. Association of built-environment, social-environment and personal factors with bicycling as a mode of transportation among Austrian city dwellers Prev. Med. (Baltim), 47 (2008), pp. 252-259 http://dx.doi.org/10.1016/j.ypmed.2008.02.019

Troped et al., 2001 P.J. Troped, R.P. Saunders, R.R. Pate, et al. Associations between self-reported and objective physical environmental factors and use of a community rail-trail Prev. Med. (Baltim), 32 (2001), pp. 191200 http://dx.doi.org/10.1006/pmed.2000.0788 
U.S. Department of Health and Human Services, 1996 U.S. Department of Health and Human Services. Physical Activity and Health: A Report of the Surgeon General. Atlanta, 1996. <http://www.cdc.gov/nccdphp/sgr/pdf/sgrfull.pdf

Wahlgren and Schantz, 2012 L. Wahlgren, P. Schantz Exploring bikeability in a metropolitan setting: stimulating and hindering factors in commuting route environments BMC Public Health, 12 (2012), p.

168 http://dx.doi.org/10.1186/1471-2458-12-168

Wegman et al., 2012 F. Wegman, F. Zhang, A. Dijkstra How to make more cycling good for road safety? Accid. Anal. Prev., 44 (2012), pp. 1929 http://dx.doi.org/10.1016/j.aap.2010.11.010

Winters et al., 2010 M. Winters, M. Brauer, E.M. Setton, et al. Built environment influences on healthy transportation choices: bicycling versus driving $\mathrm{J}$. Urban Health Bull. New York Acad. Med., 87 (2010), pp. 969$993 \mathrm{http}: / / d x$. doi.org/10.1007/s11524-010-9509-6

Winters et al., 2011 M. Winters, G. Davidson, D. Kao, et al. Motivators and deterrents of bicycling: comparing influences on decisions to ride Transportation (Amst), 38 (2011), pp. 153-168 http://dx.doi.org/10.1007/s11116010-9284-y

World Health Organisation, 2011 World Health Organisation. Guideline for Community Noise. 2011.

<http://www.who.int/docstore/peh/noise/Commnoise4.htm>. 